\title{
Four simple recommendations to encourage best practices in
}

\section{research software [version 1; peer review: 3 approved]}

Rafael C. Jiménez (D1), Mateusz Kuzak², Monther Alhamdoosh (D)3, Michelle Barker4, Bérénice Batut (i) 5, Mikael Borg6 ${ }^{6}$ Salvador Capella-Gutierrez (iD), Neil Chue Hong ${ }^{8}$, Martin Cook ${ }^{1}$, Manuel Corpas (iD), Madison Flannery ${ }^{10}$, Leyla Garcia(D11, Josep Ll. Gelpí12,13, Simon Gladman'10, Carole Goble ${ }^{14}$, Montserrat González Ferreiro 11, Alejandra Gonzalez-Beltran (iD15, Philippa C. Griffin 10, Björn Grüning (iD), Jonas Hagberg (iD6, Petr Holub16, Rob Hooft (Di) 17, Jon Ison 18, Daniel S. Katz (D19-22, Brane Leskošek23, Federico López Gómez (D1, Luis J. Oliveira24, David Mellor (iD25, Rowland Mosbergen26, Nicola Mulder (D)27, Yasset Perez-Riverol(D11, Robert Pergl28, Horst Pichler29, Bernard Pope (D)10, Ferran Sanz (D)30, Maria V. Schneider ${ }^{10}$, Victoria Stodden ${ }^{20}$, Radosław Suchecki31, Radka Svobodová Vařeková32,33, Harry-Anton Talvik ${ }^{34}$, Ilian Todorov ${ }^{35}$, Andrew Treloar ${ }^{36}$, Sonika Tyagi ${ }^{10,37}$, Maarten van Gompel ${ }^{38}$, Daniel Vaughan ${ }^{11}$, Allegra Via39, Xiaochuan Wang ${ }^{40}$, Nathan S. Watson-Haigh ${ }^{31}$, Steve Crouch ${ }^{41}$

\footnotetext{
${ }^{1}$ ELIXIR-EE, Institute of Computer Science, University of Tartu, Tartu, Estonia

${ }^{2}$ Science \& Technologies Facilities Council, Swindon, UK

${ }^{3}$ Australian National Data Service, Melbourne, Australia

${ }^{4}$ Australian Genome Research Facility Ltd., Melbourne, Australia

${ }^{5}$ Centre for Language and Speech Technology, Radboud University Nijmegen, Nijmegen, The Netherlands

${ }^{6}$ IBPM-CNR, Department of Biochemical Sciences, Sapienza University of Rome, Rome, Italy

${ }^{7}$ Faculty of Information Technology, Monash University, Victoria, Australia

${ }^{8}$ Software Sustainability Institute, Web and Internet Science, University of Southampton, Southampton, UK

${ }^{9}$ ELIXIR Hub, Wellcome Genome Campus, Hinxton, CB10 1SD, UK

${ }^{10}$ Netherlands eScience Center, Science Park 140, Amsterdam, 1098 XG, The Netherlands

${ }^{11}$ CSL Limited, Bio21 Institute, 30 Flemington Road, Parkville, Victoria, 3010, Australia

${ }^{12}$ National eResearch Collaboration Tools and Resources, Victoria, 3010, Australia

${ }^{13}$ ELIXIR-DE and de.NBI, Bioinformatics Group, Department of Computer Science, University of Freiburg, Freiburg, Germany

${ }^{14}$ ELIXIR-SE, National Bioinformatics Infrastructure Sweden (NBIS), Scilifelab, Department of Biochemistry and Biophysics (DBB), Stockholm University, Stockholm, Sweden

${ }^{15}$ ELIXIR-ES, Spanish National Bioinformatics Institute (INB), Spanish National Cancer Research Centre (CNIO), Calle de Melchor Fernández Almagro 3, Madrid, 28029, Spain

${ }^{16}$ Software Sustainability Institute, JCMB, University of Edinburgh, Edinburgh, EH9 3FD, UK

${ }^{17}$ Repositive Ltd, Future Business Centre, Cambridge, UK

${ }^{18}$ EMBL Australia Bioinformatics Resource, Lab-14, The University of Melbourne, 700 Swanston St, Parkville, Victoria, 3053, Australia

${ }^{19}$ EMBL-EBI, Wellcome Genome Campus, Hinxton, CB10 1SD, UK

${ }^{20}$ ELIXIR-SI, Faculty of Medicine, University of Ljubljana, Ljubljana, Slovenia

${ }^{21}$ DETI/IEETA, University of Aveiro, Aveiro, Portugal

${ }^{22}$ Center for Open Science, Charlottesville, VA, USA

${ }^{23}$ Stemformatics, University of Melbourne, Melbourne, Australia

${ }^{24}$ Computational Biology Division, Department of Integrative Biomedical Sciences, Institute for Infectious Disease and Molecular
} 
Medicine, University of Cape Town, Cape Town, South Africa

${ }^{25}$ ELIXIR-CZ, Faculty of Information Technology, Czech Technical University in Prague, Prague, Czech Republic

${ }^{26}$ BBMRI.at, Alpen-Adria-University Klagenfurt, Klagenfurt, Austria

${ }^{27}$ GRIB, Institut Hospital del Mar d'Investigacions Mèdiques (IMIM), Universitat Pompeu Fabra, Barcelona, Spain

${ }^{28}$ School of Agriculture, Food \& Wine, University of Adelaide, Adelaide, Australia

${ }^{29}$ Central European Institute of Technology (CEITEC), Brno, Czech Republic

${ }^{30}$ National Centre for Biomolecular Research, Masaryk University, Brno, Czech Republic

${ }^{31}$ Barcelona Supercomputing Center, Barcelona, 08034, Spain

32Department of Biochemistry and Molecular Biomedicine, Universitat de Barcelona, Barcelona, 08028, Spain

${ }^{33}$ ELIXIR-UK, Software Sustainability Institute, School of Computer Science, University of Manchester, Oxford Road, Manchester, M13

9PL, UK

${ }^{34}$ Oxford e-Research Centre, University of Oxford, Oxford, UK

${ }^{35}$ BBMRI-ERIC, Neue Stiftingtalstraße 2/B/6, Graz, 8010, Austria

${ }^{36}$ Dutch TechCenter for Life Sciences and ELIXIR-NL, Utrecht, The Netherlands

37ELIXIR-DK, Technical University of Denmark, Denmark, Denmark

${ }^{38}$ National Center for Supercomputing Applications, University of Illinois at Urbana-Champaign, Urbana, IL, USA

${ }^{39}$ School of Information Sciences, University of Illinois Urbana Champaign, Urbana, IL, USA

40Department of Electrical and Computer Engineering, University of Illinois Urbana Champaign, Urbana, IL, USA

${ }^{41}$ Department of Computer Science, University of Illinois Urbana Champaign, Urbana, IL, USA

V1 First published: 13 Jun 2017, 6:876

https://doi.org/10.12688/f1000research.11407.1

Latest published: 13 Jun 2017, 6:876

https://doi.org/10.12688/f1000research.11407.1

\section{Abstract}

Scientific research relies on computer software, yet software is not always developed following practices that ensure its quality and sustainability. This manuscript does not aim to propose new software development best practices, but rather to provide simple recommendations that encourage the adoption of existing best practices. Software development best practices promote better quality software, and better quality software improves the reproducibility and reusability of research. These recommendations are designed around Open Source values, and provide practical suggestions that contribute to making research software and its source code more discoverable, reusable and transparent. This manuscript is aimed at developers, but also at organisations, projects, journals and funders that can increase the quality and sustainability of research software by encouraging the adoption of these recommendations.

\section{Keywords}

Open Source, code, software, guidelines, best practices, recommendations, Open Science, quality, sustainability, FAIR

\section{Open Peer Review}

\begin{tabular}{lccc}
\hline Approval Status & \multicolumn{2}{c}{} \\
\hline & 1 & 2 & 3 \\
\hline version 1 & & & \\
13 Jun 2017 & view & view & view
\end{tabular}

1. Roberto Di Cosmo ID , Inria, Rocquencourt, France

Paris Diderot University, Paris, France

2. Gregory V. Wilson, Rangle.io, Toronto,

Canada

3. Stefanie Betz, Karlsruhe Institute of

Technology (KIT), Karlsruhe, Germany

Any reports and responses or comments on the article can be found at the end of the article.

This article is included in the ELIXIR gateway. 
This article is included in the Bioinformatics

gateway.

This article is included in the Bioinformatics

Education and Training Collection collection.

EMBL-EBI

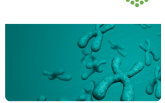

This article is included in the EMBL-EBI

collection.

Corresponding authors: Rafael C. Jiménez (rafael.jimenez@elixir-europe.org), Mateusz Kuzak (m.kuzak@esciencecenter.nl), Steve Crouch (s.crouch@software.ac.uk)

Competing interests: At the time of writing $\mathrm{MC}$ is an employee of Repositive Ltd.

Grant information: This work was partially supported by ELIXIR-EXCELERATE and CORBEL. ELIXIR-EXCELERATE and CORBEL are funded by the European Commission within the Research Infrastructures programme of Horizon 2020, grant agreement numbers 676559 and 654248. The European workshops were supported by ELIXIR and organised in collaboration with the Software Sustainability Institute and Netherlands eScience Center. The workshop in Australia was supported by EMBL-ABR via its main funders: The University of Melbourne and Bioplatforms Australia.

Copyright: ๑ 2017 Jiménez RC et al. This is an open access article distributed under the terms of the Creative Commons Attribution License, which permits unrestricted use, distribution, and reproduction in any medium, provided the original work is properly cited.

How to cite this article: Jiménez RC, Kuzak M, Alhamdoosh M et al. Four simple recommendations to encourage best practices in research software [version 1; peer review: 3 approved] F1000Research 2017, 6:876 https://doi.org/10.12688/f1000research.11407.1

First published: 13 Jun 2017, 6:876 https://doi.org/10.12688/f1000research.11407.1 


\section{Introduction}

New discoveries in modern science are underpinned by automated data generation, processing and analysis: in other words, they rely on software. Software, particularly in the context of research, is not only a means to an end, but is also a collective intellectual product and a fundamental asset for building scientific knowledge. More than $90 \%$ of scientists acknowledge software is important for their own research and around $70 \%$ say their research would not be feasible without it (Hannay et al., 2009; Hettrick et al., 2016).

Scientists are not just users of software; they are also prime producers (Goble, 2014). 90\% of scientists developing software are primarily self-taught and lack exposure and incentives to adopt software development practices that are widespread in the broader field of software engineering (Wilson et al., 2014). As a result, software produced for research does not always meet the standards that would ensure its quality and sustainability, affecting the reproducibility and reusability of research (Crouch et al., 2013).

Open Source Software (OSS) is software with source code that anyone can inspect, modify and enhance. OSS development is used by organisations and projects to improve accessibility, reproduction, transparency and innovation in scientific research (Mulgan et al., 2005; Nosek et al., 2015). OSS not only increases discoverability and visibility, but it also engages developer and user communities, provides recognition for contributors, and builds trust among users (McKiernan et al., 2016). OSS development significantly contributes to the reproducibility of results generated by the software and facilitates software reusability and improvement (Ince et al., 2012; Perez-Riverol et al., 2014). Opening code to the public is also an opportunity for developers to showcase their work, so it becomes an incentive for adoption of software development best practices (Leprevost et al., 2014). Thus, OSS can be used as a vehicle to promote the quality and sustainability of software, leading to the delivery of better research.

This manuscript describes a core set of OSS recommendations to improve the quality and sustainability of research software. It does not propose new software development best practices, but rather provides easy-to-implement recommendations that encourage adoption of existing best practices. These recommendations do not aim to describe in detail how to develop software, but rather lay out practical suggestions on top of Open Source values that go towards making research software and its source code more discoverable, reusable and transparent.

The OSS recommendations should be applied following existing and complementary guidelines like best practices, manifestos and principles that describe more specific procedures on how to develop and manage software. Some of these complementary guidelines are related to version control, code review, automated testing, code formatting, documentation, citation and usability.
(Artaza et al., 2016; DagstuhlEAS, 2017; Gilb, 1988; Leprevost et al., 2014; List et al., 2017; Perez-Riverol et al., 2016; Prlić \& Procter, 2012; Smith et al., 2016; Wilson et al., 2014; Wilson et al., 2016).

This manuscript also aims to encourage projects, journals, funders and organisations to both endorse the recommendations and to drive compliance through their software policies. The recommendations are accompanied by a list of arguments addressing common questions and fears raised by the research community when considering open sourcing software.

In this manuscript, software is broadly defined to include command line software, graphical user interfaces, desktop and mobile applications, web-based services, application program interfaces (APIs) and infrastructure scripts that help to run services.

\section{Target audience}

Our target audience includes leaders and managers of organisations and projects, journal editorial bodies, and funding agencies concerned with the provision of products and services relying on the development of open research software. We want to provide these stakeholders with a simple approach to drive the development of better software. Though these OSS recommendations have mostly been developed within, and received feedback from, the life science community, the document and its recommendations apply to all research fields.

Strategies to increase software quality usually target software developers, focusing on training and adoption of best practices (Wilson et al., 2014). This approach can yield good results, but requires a significant effort as well as personal commitment from developers (Wilson, 2014). For an organisation employing scientists and developers with different sets of programming skills and responsibilities, it is not easy to endorse specific best practices or define a broad range of training needs. It is easier to endorse a set of basic recommendations that are simple to monitor, simple to comply with, and which drive the adoption of best practices and reveal training needs. The OSS recommendations aim to create awareness, encourage developers to be more conscious of best practices, and make them more willing to collaborate and request support. The recommendations define broad guidelines, giving developers freedom to choose how to implement specific best practices.

In terms of the adoption of these recommendations, we see endorsement as the first step: that is, agreeing to support the OSS recommendations without a formal process for implementation. Promotion is a second step: that is, actively publicising and incentivising the OSS recommendations within the organisation as well as globally. Compliance is the third step: to formally implement them within the organisation, with ongoing monitoring and public reporting if possible. To facilitate progress, we propose that organisations, projects, journals, as well as funding agencies include these OSS recommendations as part of their policies relating to the development and publication of software. 
Open Source Software is not just adopted by non-profit organisations, but also by commercial companies as a business model (Popp, 2015). Therefore, we encourage not only publicly funded projects but also for-profit entities to adopt OSS and support these recommendations.

\section{Recommendations}

1. Make source code publicly accessible from day one Develop source code in a publicly accessible, version controlled repository (e.g., GitHub and Bitbucket) from the beginning of the project. The longer a project is run in a closed manner, the harder it is to open it later (Fogel, 2005). Opening code and exposing the software development life cycle publicly from day one:

- Promotes trust in the software and broader project

- Facilitates the discovery of existing software development projects

- Provides a historical public record of contributions from the start of the project and helps to track recognition

- Encourages contributions from the community

- Increases opportunities for collaboration and reuse

- Exposes work for community evaluation, suggestions and validation

- Increases transparency through community scrutiny

- Encourages developers to think about and showcase good coding practices

- Facilitates reproducibility of scientific results generated by all prior versions of the software

- Encourages developers to provide documentation, including a detailed user manual and clear in-code comments

Some common doubts and questions about making software Open Source are discussed in the Supplementary File S1, "Fears of open sourcing and some ways to handle them".

\section{Make software easy to discover by providing software} metadata via a popular community registry

Facilitate discoverability of the software project and its source code by registering metadata related to the software in a popular community registry. Metadata might include information like the source code location, contributors, licence, version, identifier, references and how to cite the software. Metadata registration:

- Increases the visibility of the project, the software, its use, its successes, its references, and its contributors

- Provides easy access for software packagers to deploy your software, thus increasing visibility

- Encourages software providers to think about the metadata that describes software as well as how to expose such metadata

- Helps to expose the software metadata in a machine readable format via the community registry

- Increases the chances of collaboration, reuse, and improvement

Examples of community registries of software metadata are bio. tools (Ison et al., 2016), (Ison et al., 2016) biojs.io (Corpas et al., 2014; Gómez et al., 2013) and Omic Tools (Henry et al., 2014) in the life sciences and DataCite (Brase, n.d.) as a generic metadata registry for software as well as data.

\section{Adopt a licence and comply with the licence of third-party} dependencies

Adopt a suitable Open Source licence to clarify how to use, modify and redistribute the source code under defined terms and conditions. Define the licence in a publicly accessible source code repository, and ensure the software complies with the licences of all third party dependencies. Providing a licence:

- Clarifies the responsibilities and rights placed on third parties wishing to use, copy, redistribute, modify and/or reuse your source code

- Enables using the code in jurisdictions where "code with no licence" means it cannot be used at all

- Protects the software's intellectual property

- Provides a model for long-term sustainability by enabling legally well-founded contributions and reuse

We advise choosing a OSI-approved Open Source Licence unless your institution or project requires a different licence. Websites like "Choose an open source license" provide guidelines to help users to select an OSI-approved Open Source Licence. Organisations like the OSS Watch also provide advice on how to keep track of the licences of software dependencies. For reusability reasons, we also advise authors to disclose any patents and pending patent applications known to them affecting the software.

\section{Define clear and transparent contribution, governance and communication processes}

Open sourcing your software does not mean the software has to be developed in a publicly collaborative manner. Although it is desirable, the OSS recommendations do not mandate a strategy for collaborating with the developer community. However, projects should be clear about how contributions can be made and incorporated by having transparent governance model and communication channels. Clarity on the project structure, as well as its communication channels and ways to contribute:

- Increases transparency on how the project and the software is being managed

- Helps to define responsibilities and how decision are made in the software project 
- Helps the community know how to collaborate, communicate and contribute to the project

For instance the Galaxy project's website describes the team's structure, how to be part of the community, and their communication channels.

\section{Alignment with FAIR data principles}

The FAIR Guiding Principles for scientific data management and stewardship provide recommendations on how to make research data findable, accessible, interoperable and reusable (FAIR) (Wilkinson et al., 2016). While the FAIR principles were originally designed for data, they are sufficiently general that their high level concepts can be applied to any digital object including software. Though not all the recommendations from the FAIR data principles directly apply to software, there is good alignment between the OSS recommendations and the FAIR data principles (see Table 1).
There are also distinctions between the OSS recommendations and the FAIR data principles. The FAIR data principles have a specific emphasis on enhancing machine-readability: the ability of machines to automatically find and use data. This emphasis is not present in the OSS recommendations which expect machine readable software metadata to be available via software registries. The OSS recommendations are less granular and aim to enhance understanding and uptake of best practices; they were designed with measurability in mind. The FAIR data principles do not have such built-in quantification yet. FAIR metrics are a separate effort under development, lead by the Dutch Techcentre for Life Sciences (Eijssen et al., 2016).

The community registries can play an important role in making software metadata FAIR by capturing, assigning and exposing software metadata following a standard knowledge representation and controlled vocabularies that are relevant for domain-specific communities. Thus we expect the community registries to provide

Table 1. Comparison between the OSS recommendations and the FAIR data principles (Wilkinson et al., 2016).

\section{The FAIR Guiding Principles}

To be Findable: F1. (meta)data are assigned a globally unique and persistent identifier; F2. data are described with rich metadata (defined by R1 below); F3. metadata clearly and explicitly include the identifier of the data it describes; F4. (meta)data are registered or indexed in a searchable resource

To be Accessible: A1. (meta)data are retrievable by their identifier using a standardized communications protocol; A1.1 the protocol is open, free, and universally implementable; A1.2 the protocol allows for an authentication and authorization procedure, where necessary; A2. metadata are accessible, even when the data are no longer available

To be Interoperable: 11. (meta)data use a formal, accessible, shared, and broadly applicable language for knowledge representation; 12. (meta)data use vocabularies that follow FAIR principles; I3. (meta)data include qualified references to other (meta)data

To be Reusable: R1. meta(data) are richly described with a plurality of accurate and relevant attributes; R1.1. (meta)data are released with a clear and accessible data usage license: R1.2. (meta)data are associated with detailed provenance; R1.3. (meta)data meet domain-relevant community standards

\section{OSS recommendations}

"R2. Make software easy to discover by providing software metadata via a popular community registry" aligns with the Findability principle, helping to increase visibility and helping software providers to think about how to describe software metadata (versions, identifiers, contributors, citations, etc.)

"R1. Make source code publicly accessible from day one" focuses on openness including accessibility. The FAIR accessible principle instead opens the door to data that is restricted access e.g. for privacy reasons. Since such reasons do not apply for software, the OSS recommendations prefer to direct towards openness instead, supporting open science to the maximum extent.

This OSS recommendations do not aim to address software interoperability directly but contribute to a more homogenous description of software by encouraging software providers to register software metadata into registries providing specific metadata guidelines.

"R3. Adopt a license and comply with the licence of third-party dependencies" aligns with the Reusability principle, helping to define to what extent the source code can be used and reused by the community, as a standalone software or as part of other software.

Open availability of tools and libraries working with data formats can be a great help in making data interoperable: e.g. reuse of the same tools to read and write data can prevent subtle interoperability problems.

Reproducibility of experiments and reuse of data is facilitated by the open availability of the associated software which is part of the provenance. All of the OSS recommendations thereby facilitate data Reusability. 
guidelines on how to provide software metadata following the FAIR Guiding Principles (Wilkinson et al., 2016).

\section{Conclusion}

The OSS recommendations aim to encourage the adoption of best practices and thus help to develop better software for better research. These recommendations are designed as practical ways to make research software and its source code more discoverable, reusable and transparent, with the desired objective to improve its quality and sustainability. Unlike many software development best practices tailored for software developers, the OSS recommendations aim to target a wider audience, particularly research funders, research institutions, journals, group leaders, and managers of projects producing research software. The adoption of these recommendations offer a simple mechanism for these stakeholders to promote the development of better software and an opportunity for developers to improve and showcase their software development skills.

\section{Author contributions}

Steve Crouch, Neil Chue Hong, Mateusz Kuzak, Manuel Corpas, Jason Williams, Maria Victoria Schneider and Rafael C Jimenez also contributed organising and facilitating workshops. Federico Lopez developed the reference website to provide information and a point of contact for these recommendations. All the authors contributed providing feedback to shape this manuscript and recommendations.

\section{Competing interests}

At the time of writing MC is an employee of Repositive Ltd.

\section{Grant information}

This work was partially supported by ELIXIR-EXCELERATE and CORBEL. ELIXIR-EXCELERATE and CORBEL are funded by the European Commission within the Research Infrastructures programme of Horizon 2020, grant agreement numbers 676559 and 654248 . The European workshops were supported by ELIXIR and organised in collaboration with the Software Sustainability Institute and Netherlands eScience Center. The workshop in Australia was supported by EMBL-ABR via its main funders: The University of Melbourne and Bioplatforms Australia.

\section{Acknowledgments}

The authors wish to thank all the supporters of the OSS recommendations.

The OSS recommendations presented in this manuscript have been have been open for discussion for more than a year. This allowed them to be developed by a wide range of stakeholders, including developers, managers, researchers, funders and project coordinators and anybody else concerned with the production of quality software for research. We also organised several workshops and presented this work in several meetings to engage more stakeholders, collect feedback and refine the recommendations. For further information, about the OSS recommendations please visit the following site: https://SoftDev4Research.github.io/ recommendations/

\section{Supplementary material}

Supplementary File 1: 'Fears of open sourcing and some ways to handle them'. In this appendix we aim to expose some of the common fear scenarios related to open sourcing, and some ways to handle them.

Click here to access the data.

Artaza $\mathrm{H}$, Chue Hong N, Manuel C, et al:: Top 10 metrics for life science software good practices [version 1; referees: 2 approved]. F1000Res. 2016; 5 : pii: ELIXIR-2000.

PubMed Abstract | Publisher Full Text | Free Full Text

Brase J: Datacite - A Global Registration Agency for Research Data. SSRN Electronic Journal. n.d.

Publisher Full Text

Corpas M, Jimenez R, Carbon SJ, et al.: BioJS: an open source standard for biological visualisation - its status in 2014 [version 1; referees: 2 approved]. F1000Res. 2014; 3: 55

PubMed Abstract | Publisher Full Text | Free Full Text

Crouch S, Chue Hong N, Hettrick S, et al.: The Software Sustainability Institute: Changing Research Software Attitudes and Practices. Computing in Science \& Engineering. 2013; 15(6): 74-80.

Publisher Full Text

DagstuhIEAS: DagstuhIEAS/draft-Manifesto. GitHub. 2017. Accessed January 5. Reference Source
Eijssen L, Evelo C, Kok R, et al:: The Dutch Techcentre for Life Sciences: Enabling data-intensive life science research in the Netherlands [version 2 referees: 2 approved, 1 approved with reservations]. F1000Res. 2016; 4: 33. PubMed Abstract | Publisher Full Text | Free Full Text

Fogel K: Producing Open Source Software: How to Run a Successful Free Software Project. O'Reilly Media, Inc. 2005.

Reference Source

Gilb T: Principles of Software Engineering Management. Addison-Wesley

Professional, 1988

Reference Source

Goble C: Better Software, Better Research. IEEE Internet Computing. 2014;

18(5): 4-8.

Publisher Full Text

Gómez J, García LJ, Salazar GA, et al:: BioJS: An Open Source JavaScript Framework for Biological Data Visualization. Bioinformatics. 2013; 29(8):

1103-4.

PubMed Abstract | Publisher Full Text | Free Full Text 
Hannay JE, MacLeod C, Singer J, et al.: How Do Scientists Develop and Use Scientific Software? In 2009 ICSE Workshop on Software Engineering for Computational Science and Engineering. 2009.

Publisher Full Text

Henry VJ, Bandrowski AE, Pepin AS, et al.: OMICtools: An Informative Directory for Multi-Omic Data Analysis. Database (Oxford). 2014; 2014: pii: bau069. PubMed Abstract | Publisher Full Text | Free Full Text

Hettrick A, Chue Hong C, De Roure C, et al.: UK Research Software Survey 2014. Accessed November 27. 2016.

Publisher Full Text

Ince DC, Hatton L, Graham-Cumming J: The Case for Open Computer Programs. Nature. 2012; 482(7386): 485-88.

PubMed Abstract | Publisher Full Tex

Ison J, Rapacki K, Ménager H, et al.: Tools and Data Services Registry: A Community Effort to Document Bioinformatics Resources. Nucleic Acids Res. 2016; 44(D1): D38-47.

PubMed Abstract | Publisher Full Text | Free Full Text

Leprevost Fda V, Barbosar VC, Francisco EL, et al.: On best practices in the development of bioinformatics software. Front Genet. 2014; 5: 199.

PubMed Abstract | Publisher Full Text | Free Full Text

List M, Ebert P, Albrecht F: Ten Simple Rules for Developing Usable Software in Computational Biology. PLoS Comput Biol. 2017; 13(1): e1005265. PubMed Abstract | Publisher Full Text | Free Full Text

McKiernan EC, Bourne PE, Brown CT, et al:: How open science helps researchers succeed. eLife. 2016; 5: pii: e16800.

PubMed Abstract | Publisher Full Text | Free Full Text

Mulgan G, Steinberg T, Salem O: Wide Open: Open Source Methods and Their Future Potential. Demos Medical Publishing. 2005.

Reference Source

Nosek BA, Alter G, Banks GC, et al.: SCIENTIFIC STANDARDS. Promoting an open research culture. Science. American Association for the Advancement of
Science, 2015; 348(6242): 1422-25.

PubMed Abstract | Publisher Full Text | Free Full Text

Perez-Riverol Y, Gatto L, Wang R, et al: Ten Simple Rules for Taking Advantage of Git and GitHub. PLoS Comput Biol. 2016; 12(7): e1004947.

PubMed Abstract | Publisher Full Text | Free Full Text

Perez-Riverol Y, Wang R, Hermjakob $\mathrm{H}$, et al.: Open source libraries and

frameworks for mass spectrometry based proteomics: a developer's

perspective. Biochim Biophys Acta. 2014; 1844(1 Pt A): 63-76.

PubMed Abstract | Publisher Full Text | Free Full Text

Popp KM: Best Practices for Commercial Use of Open Source Software:

Business Models, Processes and Tools for Managing Open Source Software.

BoD - Books on Demand. 2015.

Reference Source

Prlic A, Procter JB: Ten simple rules for the open development of scientific software. PLoS Comput Biol. 2012; 8(12): e1002802.

PubMed Abstract | Publisher Full Text | Free Full Tex

Smith AM, Katz DS, Niemeyer KE, et al.: Software Citation Principles.

PeerJ Comput Sci. 2016; 2: e86.

Publisher Full Text

Wilkinson MD, Dumontier M, Aalbersberg IJ, et al:: The FAIR Guiding Principles for scientific data management and stewardship. Sci Data. 2016; 3: 160018 PubMed Abstract | Publisher Full Text | Free Full Text

Wilson G, Aruliah DA, Brown CT, et al:: Best practices for scientific computing PLoS Biol. 2014; 12(1): e1001745.

PubMed Abstract | Publisher Full Text | Free Full Text

Wilson G, Bryan J, Cranston K, et al:: Good Enough Practices in Scientific

Computing. 2016.

Reference Source

Wilson G: Software Carpentry: lessons learned [version 1; referees: 3

approved]. F1000Res. 2014; 3: 62 .

PubMed Abstract | Publisher Full Text | Free Full Text 


\section{Open Peer Review}

\section{Current Peer Review Status:}

\section{Version 1}

Reviewer Report 10 July 2017

https://doi.org/10.5256/f1000research.12314.r23464

(C) 2017 Betz S. This is an open access peer review report distributed under the terms of the Creative Commons Attribution License, which permits unrestricted use, distribution, and reproduction in any medium, provided the original work is properly cited.

\section{Stefanie Betz}

Karlsruhe Institute of Technology (KIT), Karlsruhe, Germany

The article advocates research software openness presenting four recommendations to improve research software visibility, re-usability, and transparency. I really like the article and I think it is important to open research software. Please find below my feedback. (Overall, I agree with the comments of Milad Miladi):

In my opinion, the title does not reflect the content of the article. The focus is on adopting OSS and supporting the provided four recommendations to help to develop better software for better research. Currently, only the second part (supporting the provided four recommendations to help to develop better software for better research) is reflected in the title not the part about OSS.

I am not sure everybody is familiar with the open registry platforms. Thus, some more information regarding them or a link to background information would be nice.

I think recommendation four should include documentation.

Is the topic of the opinion article discussed accurately in the context of the current literature?

Yes

Are all factual statements correct and adequately supported by citations? Yes

Are arguments sufficiently supported by evidence from the published literature? Yes

Are the conclusions drawn balanced and justified on the basis of the presented arguments? Yes

Competing Interests: No competing interests were disclosed. 
Reviewer Expertise: Software Engineering

I confirm that I have read this submission and believe that I have an appropriate level of expertise to confirm that it is of an acceptable scientific standard.

Reviewer Report 26 June 2017

https://doi.org/10.5256/f1000research.12314.r23467

(C) 2017 Wilson G. This is an open access peer review report distributed under the terms of the Creative Commons Attribution License, which permits unrestricted use, distribution, and reproduction in any medium, provided the original work is properly cited.

\section{Gregory V. Wilson}

Rangle.io, Toronto, ON, Canada

The article presents focused, well-argued advocacy for improving software development practices in the sciences. None of the recommendations will be surprising to those already involved in open science, but as only a small minority of researchers actually do them, it is worth presenting them forcefully and succinctly. I would recommend shortening the introductory material (sections "Introduction" and "Target Audience"), but that is a minor point.

Is the topic of the opinion article discussed accurately in the context of the current literature?

Yes

Are all factual statements correct and adequately supported by citations? Yes

Are arguments sufficiently supported by evidence from the published literature? Yes

Are the conclusions drawn balanced and justified on the basis of the presented arguments? Yes

Competing Interests: No competing interests were disclosed.

Reviewer Expertise: software engineering education

I confirm that I have read this submission and believe that I have an appropriate level of expertise to confirm that it is of an acceptable scientific standard.

Reviewer Report 26 June 2017

https://doi.org/10.5256/f1000research.12314.r23468 
(C) 2017 Di Cosmo R. This is an open access peer review report distributed under the terms of the Creative Commons Attribution License, which permits unrestricted use, distribution, and reproduction in any medium, provided the original work is properly cited.

\author{
Roberto Di Cosmo \\ 1 Software Heritage, Inria, Rocquencourt, France \\ 2 Paris Diderot University, Paris, France
}

This article presents four simple recommendations that may improve the overall quality and visibility of research software. This reviewer agrees with the basic principles set forth by the authors, and hopes they will be widely shared and adopted at least for software that is expected to last longer than the time it takes for the corresponding research paper to be accepted and/or presented.

Is the topic of the opinion article discussed accurately in the context of the current literature?

Yes

Are all factual statements correct and adequately supported by citations? Yes

Are arguments sufficiently supported by evidence from the published literature? Yes

Are the conclusions drawn balanced and justified on the basis of the presented arguments? Yes

Competing Interests: No competing interests were disclosed.

Reviewer Expertise: formal methods, software engineering, programming languages, logics, theoretical computer science, semantics, parallel programming, open source software, package management, archival

I confirm that I have read this submission and believe that I have an appropriate level of expertise to confirm that it is of an acceptable scientific standard.

\title{
Comments on this article
}

\section{Version 1}

Reader Comment 02 Aug 2019

Teresa Gomez-Diaz, CNRS/LIGM, University of Paris-Est Marne la Vallée, France 
Very interesting article! Research software importance and recognition is increasing rapidly in the scientific landscape and this work provides relevant recommendations to be followed by research software producers in order to improve the disseminations practices.

The article proposals have some common points with the dissemination procedure that I described in the preprint:

"Free software, Open source software, licenses. A short presentation including a procedure for research software and data dissemination" (September 2014), https://zenodo.org/record/11709/ It was presented at the EGI Conference 2015, in the session: Workshop on open licenses: Data licencing and policies https://indico.egi.eu/indico/event/2452/session/75

Competing Interests: As competing interest, I have collaborated with one of the authors in the last four years (grant application).

\section{Reader Comment 20 Jul 2017}

\section{Paul Kersey}

I think this is an important paper, and I broadly agree with its recommendations, but it could do (IMO) with a little copy-editing before it is distributed to funding agencies and other people identified as among its target audience. I'm writing these comments here at the encouragement of the lead author.

A.TITLE

I don't think the title is quite right. The paper recommends practices for the development of best software, i.e. (i) open sourcing of code is a practice, not a means to encourage a practice (ii) the adoption of practices (whatever they are) the means not the end I would suggest "Good Practices in Research Software" as a better title: 5 words not 10, and the dropped words just obfuscate.

\section{B.RECOMMENDATION 2}

"Make software easy to discover by providing software metadata via a popular community registry" - what matters is that the registry is popular. What is meant by a community registry? Why it is important that a registry is run by a community? Wouldn't you prefer a Google-run registry?

\section{C.CONCLUSIONS}

"The OSS recommendations aim to encourage the adoption of best practices (1) and thus help to develop better software for better research (2). These recommendations are designed as practical ways to make research software and its source code more discoverable, reusable and transparent, with the desired objective to improve its quality and sustainability (3). Unlike many software development best practices tailored for software developers, the OSS recommendations aim to target a wider audience, particularly research funders, research institutions, journals, group leaders, and managers of projects producing research software (4). The adoption of these recommendations offer a simple mechanism for these stakeholders to promote the development 
of better software and an opportunity for developers to improve and showcase their software development skills (5)."

1.This amounts to saying "We recommend that people follow these recommendations". The recommendations are recommendations of good practice. These practices are recommended with the aim of doing something - that aim is not to encourage their own adoption!

2.Better software or better research? How is the research better? You address this in the introduction but the treatment here is a bit glib.

3. The structure of this sentence - "we do A to do B to do C" - makes it unclear what the ultimate goal is, and the individual links in the logic chain are unsupported. I think the way I would put it is: "Research software is often tapped in a vicious cycle: small user communities can sustain only a limited effort in maintenance, development and documentation. We believe that an open source approach, as recommended here, can help to overcome these problems".

4. "Unlike many software development best practices tailored for software developers, the OSS recommendations aim to target a wider audience, particularly research funders, research institutions, journals, group leaders, and managers of projects producing research software." "Aiming to target" is a neoplasm. Also, the "unlike" is a bit tenuous - all development practices are tailored for developers (or else they're a bit silly), but obviously managers are also generally interested and involved. I think the point is simply "We urge not only software developers but also research funders, research institutions, journals, group leaders, and managers of projects producing research software, to consider these recommendations".

5. The last sentence is a bit weak as a conclusion. There's a powerful point (which is well-made in the introduction) that open source software can be validated, improved upon, and used to enable research; that the culture of open source promotes the development of both the coder and the code; and that the spoils of publicly-funded tool development properly belong in the public domain. But instated, the final conclusion is a piece of career advice, and moreover, amounts to saying "people should follow these recommendations because they are good", rather than demonstrating why they are good.

Competing Interests: No competing interests were disclosed.

\section{Reader Comment 15 Jun 2017}

\section{Milad Miladi}

Nice work! I really appreciate the authors effort to encourage and raise the importance of opening the research software. Here are my comments about the manuscript:

* The title does not reflect well the aim and scope of the article. Throughout the text reader would realize focus on the importance of open source software and the aim is to encourage and guide principle investigators, managers, etc for opening and managing OSS projects. From the title 
"openness" and "encouragement" is not understood.

* Although the importance of documentation has been stated in numerous previous works, it worth to be mentioned here again. Specially in my opinion this meta-data should be available independent and in parallel to of the availability via community registry.

* From the text the reader may not be fully convinced about the advantages of using open registry platforms over plain text format beside the source code. A brief overview of the mentioned platforms (bio.tools,..) like number of current entries, the differences, ... would be nice.

* Reusability and reproducibility of the research tools are among major concerns of nowadays research ecosystem. This has been stated in the abstract and introduction sections, however it would be very valuable if the authors could include specific recommendations on how these metrics can be improved for research softwares.

Competing Interests: No competing interests were disclosed.

\section{Reader Comment 13 Jun 2017}

\section{Mehran Karimzadeh}

Very interesting work. You may be interested in our previous perspective, "Top considerations for creating bioinformatics software documentation" (https://doi.org/10.1093/bib/bbw134), which might be a useful reference in the part of your article that mentions existing guidelines in documentation and elsewhere.

Competing Interests: No competing interests were disclosed. 
The benefits of publishing with F1000Research:

- Your article is published within days, with no editorial bias

- You can publish traditional articles, null/negative results, case reports, data notes and more

- The peer review process is transparent and collaborative

- Your article is indexed in PubMed after passing peer review

- Dedicated customer support at every stage

For pre-submission enquiries, contact research@f1000.com 